\title{
Effects on health-related quality of life in patients treated with lurasidone for bipolar depression: results from two placebo controlled bipolar depression trials
}

Krithika Rajagopalan ${ }^{1}$, Elizabeth Dansie Bacci², Daisy Ng-Mak ${ }^{1}$ Kathy Wyrwich³, Andrei Pikalov ${ }^{1}$ and Antony Loebel ${ }^{1}$

\begin{abstract}
Background: Depressive symptoms associated with bipolar disorder negatively impact health-related quality of life (HRQOL). The efficacy of lurasidone in reducing depressive symptoms has been previously demonstrated. The objective of this study was to examine the direct and indirect effect (mediated through improvement in depression symptoms) of lurasidone in improving patient HRQoL.

Methods: A secondary analysis of data was conducted of two 6-week, double-blind, placebo-controlled trials assessing the effect of lurasidone (lurasidone monotherapy [20-60 mg/day or 80-120 mg/day]; lurasidone adjunctive to lithium or valproate [20-120 mg/day]) in patients with bipolar depression. Patient HRQoL was measured using the Quality of Life Enjoyment and Satisfaction Questionnaire Short Form (Q-LES-Q SF). Depression symptoms were measured using the Montgomery-Asberg Depression Rating Scale (MADRS). Analysis of covariance (ANCOVA) was used to estimate the effect of lurasidone on improvement in the Q-LES-Q SF percentage maximum score from baseline to 6 weeks. Path analysis was used to evaluate the total effect $\left(\beta_{1}\right)$, as well as the indirect $\left(\beta_{2}{ }^{*} \beta_{3}\right)$ and direct $\left(\beta_{4}\right)$ effect of lurasidone on Q-LES-Q SF change through improvements in MADRS.
\end{abstract}

Results: A total of 340 and 485 patients from the monotherapy and adjunctive therapy, respectively, were included in the analysis. At 6-weeks, ANCOVA analyses demonstrated that lurasidone provided significant improvement in adjusted mean Q-LES-Q SF scores in comparison to placebo for monotherapy (22.9 and 22.7 vs. 15.2, both $p<0.01$ ) and adjunctive therapy (23.1 vs. 17.9, $p=0.01$ ). Path analyses indicated that lurasidone treatment predicted MADRS improvement (monotherapy: $\beta_{2}=-0.44, p<0.001$; adjunctive therapy: $\beta_{2}=-0.34, p=0.003$ ), which subsequently predicted improvement in Q-LES-Q SF (monotherapy: $\beta_{3}=-0.73, p<0.001$; adjunctive therapy: $\beta_{3}=-0.75, p<0.001$ ); however, the effect of lurasidone on improvement in Q-LES-Q SF was largely mediated by change in MADRS (monotherapy: $\beta_{4}=0.11, p=0.13$; adjunctive therapy: $\beta_{4}=0.02, p=0.77$ ).

Conclusions: Lurasidone as a monotherapy and adjunctive to lithium or valproate is an effective treatment for improving HRQoL in patients with bipolar depression. However, improvement in HRQoL was not independent of improvement in depression, indicating that the effect of lurasidone on improving patient HRQoL may act through a reduction in depressive symptoms associated with bipolar disorder.

Trial registration: Clinicaltrials.gov identifiers: NCT00868699 and NCT00868452 (both registered March 23, 2009)

Keywords: Quality of life enjoyment and satisfaction questionnaire, Patient functioning, Bipolar disorder, Depression, Drug therapy

\footnotetext{
* Correspondence: elizabeth.bacci@evidera.com

2Evidera, Seattle, WA, USA

Full list of author information is available at the end of the article
} 


\section{Background}

Bipolar disorder is a chronic and debilitating mental illness, characterized by recurrent mood fluctuations between periods of depressive and manic symptoms, with an estimated lifetime prevalence of $1.0 \%[1,2]$ and is the $12^{\text {th }}$ leading cause of disability worldwide [3]. Frequent episodes of depression represent the most common symptomatic state in individuals with bipolar disorder $[4,5]$, where depressive episodes are associated with a greater negative impact on social, occupational, and general functioning than episodes of mania [4, 6-8]. Consequently, health-related quality of life (HRQoL) is also highly compromised in individuals with bipolar depression, where patients with bipolar depression report even greater worsening in their HRQoL and other indicators of functioning than patients with other mood disorders or mental illnesses $[4,9,10]$.

Traditionally, clinical trials of treatment efficacy in patients with bipolar depression have focused on symptom remission (using the Diagnostic and Statistical Manual-IV-TR [DSM-IV-TR] or clinician-reported outcome assessments such as the Montgomery-Asberg Depression Rating Scale [MADRS] score $\leq 10$ to signify remission status [11]). Increasingly, the ability of the patient to perform activities of daily living, engage and nurture social relationships, and function independently are being considered when evaluating treatment efficacy $[4,12,13]$, as the burden of bipolar depression can persist even in patients considered to be in symptomatic remission [14, 15]. Given this, HRQoL assessments can provide an additional indicator of improvement in areas valued by the patient beyond symptomatic improvement [16].

Relatively few clinical trials have incorporated patientreported outcome (PRO) measures to assess improvement in HRQoL in drug registration trials, although the number of studies incorporating these tools is increasing given the increasingly holistic view of improving patient's lives in treating chronic illnesses such as bipolar disorder [4, 17]. A literature review conducted by Revicki et al. in 2005 suggested that HRQoL may improve as a result of treatment, although these studies were limited to a short follow-up duration and small sample size [18]. More recently, IsHak et al. [19] expanded on previous reviews to address if various forms of treatment (e.g., drug therapy, behavioral therapy) have an impact on bipolar disorder, demonstrating that several drug and psychotherapeutic treatment regimens were associated with improved HRQoL in this patient population.

Only two medications were approved for the treatment of acute bipolar depression as a monotherapy in the United States until 2013 (e.g., combination of olanzapine-fluoxetine and quetiapine) [20-22]. In two placebo-controlled clinical trials, the atypical antipsychotic lurasidone, approved by the US FDA as a monotherapy and adjunctive to lithium or valproate, was found to significantly improve symptoms of depression, HRQoL, and other PROs in patients with bipolar depression over a 6 -week period [23, 24]. Thus, positive benefits in areas of life valued by the patient were achieved over the trial period. However, it is important to know if PRO improvements were a direct result of lurasidone treatment (a direct effect), or if this HRQoL improvement is achieved due to reductions in symptoms of depression (a completelymediated indirect effect) or a combination of both, direct and indirect effects. With this information, clinicians and payors will have a much greater understanding of the total effects associated with lurasidone treatment on HRQoL. Thus, the aim of this study was to examine the direct and indirect effects (mediated through improvement in bipolar depression symptoms) of lurasidone in improving patient HRQoL.

\section{Methods \\ Study design and data source}

A secondary analysis of data from two randomized, 6-week, multicenter, double-blind, placebo-controlled, parallel-group studies conducted to assess the efficacy of lurasidone as a treatment for bipolar depression (NCT00868699 and NCT00868452) were used to assess the direct and indirect effects of lurasidone on HRQoL improvement. The study population in this analysis was the intention-to-treat (ITT) population. For monotherapy, the ITT population consisted of a total of 505 randomized patients receiving at least one dose of study medication at doses of $20-60 \mathrm{mg} /$ day or $80-120 \mathrm{mg} /$ day, with at least one post-baseline efficacy measurement for the MADRS. Following a washout period of at least 3 days, patients were randomly assigned in a 1:1:1 ratio via an interactive voice response system to receive 6 weeks of treatment with lurasidone, at flexible daily doses of either $20-60 \mathrm{mg}$ or 80-120 mg, or 6 weeks of placebo. For adjunctive therapy, the ITT population consisted of a total of 672 randomized patients receiving at least one dose of study medication at a dose of 20-120 mg/day adjunctive to lithium or valproate, with at least one post-baseline efficacy measurement for the MADRS. Patients underwent stratified randomization, based on treatment with either lithium or valproate, to either adjunctive lurasidone 20$120 \mathrm{mg} /$ day or placebo in a 1:1 ratio via an interactive voice response system. The dose of mood stabilizer was adjusted to maintain a serum level in the range of 0.6$1.2 \mathrm{mEq} /$ liter for lithium or 50-125 for valproate throughout the study.

The patient population in both studies was adult patients ( $\geq 18$ years old) with bipolar I disorder. All patients were experiencing a major depressive episode (DSM-IV-TR criteria, $\geq 4$ weeks and $<12$ months in duration), with or 
without rapid cycling, without psychotic features, and with a history of at least one lifetime bipolar manic or mixed manic episode. Diagnosis was confirmed by the MiniInternational Neuropsychiatric Interview [25] and the Bipolarity Index [26]. A MADRS [11] score $\geq 20$ and a Young Mania Rating Scale (YMRS) score $\leq 12$ were required at both screening and baseline. For both studies, efficacy assessments were obtained at baseline and weekly intervals. The primary efficacy endpoint was the mean change from baseline to week 6 in MADRS total score. At each study visit, a qualified site-based rater conducted the MADRS assessment; a second MADRS assessment was administered and scored by computer as part of a quality control process. Additional study design details, including a comprehensive list of patient entry criteria, as well as primary efficacy and safety analyses, have been published elsewhere [23, 24].

The study was approved by an institutional review board at each investigational site (from January 2009 to May 2010) and was conducted in accordance with the International Conference on Harmonisation Good Clinical Practices guidelines and with the ethical principles of the Declaration of Helsinki. Written informed consent was obtained from all participants prior to participation.

\section{Scales and assessments included in this analysis Symptom assessment}

Montgomery Asberg Depression Rating Scale (MADRS)

The patient's primary bipolar depression symptoms were assessed using the MADRS, a clinician-administered depression rating scale developed from a larger scale to be sensitive to change [11]. The MADRS has 10 items, each scored on a 0-6 scale. A score of 0 indicates an absence of that symptom, and anchor point descriptors are given for scores of $0,2,4$, and 6 . Items assess many facets of depression, including sadness, tension, pessimism, suicidal thoughts, reduced sleep, and reduced appetite. A total score of 60 is possible, indicating greater depression severity.

\section{Quality of life enjoyment and satisfaction questionnaire short form}

The Q-LES-Q SF is a 16-item HRQoL measure of the degree of enjoyment and satisfaction experienced by patients in various areas of daily living [27]. The 16 items reduce to eight summary scales that reflect major areas of functioning: physical health, mood, leisure time activities, social relationships, general activities, work, household duties, and school/coursework. Each item is rated on a 5 -point scale, ranging from 1 (very poor) to 5 (very good). The first 14 items are the same as the General Activities section of the regular Q-LES-Q form and are used to compute the raw score (Items 15 and 16 were not used in the present analyses). The sum of scores for items 1-14 can range from 14-70, and is expressed as a percentage $(0-100)$ of the maximum total score that is achievable. Higher scores indicate better HRQoL.

The demographic and baseline characteristics of all participants were summarized using descriptive statistics (mean, standard deviation, range, frequencies for categorical variables). Descriptive statistics for the Q-LESQ SF and the MADRS were summarized at baseline. All analyses were stratified by clinical trial (i.e., monotherapy and adjunctive therapy).

\section{Efficacy analyses}

For the current analysis, mean change in Q-LES-Q SF percentage maximum scores $(\triangle \mathrm{Q}$-LES-Q SF) from baseline to 6 weeks was used to assess the efficacy of treatment on HRQoL. Analysis of covariance analyses (ANCOVA) were conducted, with fixed effects for treatment group, pooled study center, and baseline score entered as covariates. Pairwise comparisons between means were performed using Scheffe's test adjusting for multiple comparisons. Model effect estimates, standard errors (SE), and $p$-values are presented for all analyses.

\section{Path analysis}

Path analysis was conducted for monotherapy and adjunctive therapy trials (collapsing across dosage groups) to assess the relationship between treatment and $\Delta \mathrm{Q}$ LES-Q SF directly and through $\triangle$ MADRS, following the procedures described by Baron and Kenny [28]. The Baron and Kenny mediation model assesses the degree of the treatment effect upon a response variable in the presence of another variable (i.e., the mediating variable). This approach allows the examination of the degree of mediation (either as partial or complete mediation), through a series of four models. Statistically significant $(p<0.05)$ effects must be obtained in Models 1 , 2 , and 3 in order to test the full mediation model in Model 4. Following this approach, four models were estimated: (1) effect of treatment on $\triangle \mathrm{Q}$-LES-Q SF; (2) effect of treatment on $\triangle$ MADRS; (3) effect of $\triangle$ MADRS on $\triangle$ Q-LES-Q SF, independent of treatment; and (4) effect of treatment $\triangle \mathrm{Q}-\mathrm{LES}-\mathrm{Q}$ SF, even when controlling for the effect of $\triangle$ MADRS. $\triangle$ MADRS would be considered a partial mediator on the relationship between lurasidone and $\triangle \mathrm{Q}$-LES-Q SF if statistically significant effects were achieved in all 4 models. Complete mediation would be indicated if the effect of treatment on $\triangle$ Q-LES-Q SF described in Model 4 was 0 . Although the Baron and Kenny model was originally implemented using ordinary least squares regression, modern path analysis methods were utilized in the current study 
following the Baron and Kenny steps in order to accurately estimate the significance of the indirect effect.

Mplus statistical software version 7.0 [29] was used to conduct all analyses for Models 1-4. The total effect $\left(\beta_{1}\right)$ of the relationship between treatment and $\Delta \mathrm{Q}-\mathrm{LES}-\mathrm{Q}$ SF was estimated in Model 1 . The direct effect of treatment on the $\triangle \mathrm{Q}$-LES-Q SF controlling for $\triangle$ MADRS $\left(\beta_{4}\right)$, and the indirect effect, were estimated in Model 4. The indirect effect was calculated as the product of the relationship between treatment and $\triangle \mathrm{Q}-\mathrm{LES}-\mathrm{Q}$ SF $\left(\beta_{2}\right)$, and the relationship between $\triangle$ MADRS to $\triangle \mathrm{Q}-\mathrm{LES}-\mathrm{Q}$ SF $\left(\beta_{3}\right)$. Bias-corrected bootstrapping was used to estimate the standard errors for the estimation of the $p$-values to determine significance of each path, as well as the indirect effect. The proportion of the effect that was mediated was calculated as $\beta_{2}^{*} \beta_{3} / \beta_{1}$, while the percentage of total variance explained by each path was reported using the standardized $R^{2}$ values. The strength of the parameter estimates was interpreted using Kenny recommendations for effect size estimates of small (0.02), medium (0.15), and large (0.40) [30] for large samples with adequate power for detecting an effect. For the analysis, each of the models controlled for baseline score of the dependent variable (i.e., Q-LES-Q SF or MADRS). Parameter estimates with corresponding p-values were calculated for all four models for both trials.

Overall model fit was assessed using various global fit indices, where the following indices and fit values were used as criteria to assess acceptable model fit: chi-square test of overall model fit; Root Mean Square Error of Approximation $($ RMSEA) $<0.06$ [31]; and Tucker Lewis Index (TLI) and the Comparative Fit Index (CFI) $>0.90$ [32].

\section{Results}

\section{Demographics and baseline characteristics}

In the monotherapy trial, 818 patients were screened, of whom $505(61.7 \%)$ were randomly assigned to 6 weeks of treatment and 485 (96.0\%) were included in the ITT population. In the adjunctive therapy trial, a total of 672 patients were screened, of whom $348(51.8 \%)$ were randomly assigned to 6 weeks of treatment and 340 $(97.7 \%)$ were included in the ITT population. Study completion rates were similar for treatment and placebo groups for monotherapy $(74 \%, 73 \%$, and $75 \%)$ and adjunctive therapy $(78.1 \%$ and $82.4 \%)$ trials, respectively, in addition to baseline demographic and clinical characteristics for both studies (Table 1).

\section{Efficacy results}

Descriptive statistics for the Q-LES-Q SF at baseline and at 6 weeks are presented in Tables 2,3, and 4, along with the corresponding change scores. For the monotherapy trial, change scores on the Q-LES-Q SF indicating improvement was significantly larger for both lurasidone $20-60 \mathrm{mg} /$ day (mean change $=21.9$ ) and $80-120 \mathrm{mg} /$ day (mean change $=23.0$ ) groups in comparison to placebo (mean change $=14.5$ ). Similar change was demonstrated for adjunctive therapy for the lurasidone group (mean change $=23.3$ ) in comparison to the placebo group (mean change $=17.9$ ) .

In the ANCOVA analyses controlling for pooled study center and baseline Q-LES-Q SF percentage maximum score for the monotherapy trial, the least squares mean (SE) change from baseline to 6 weeks on the Q-LES-Q SF was significantly greater for lurasidone $20-60 \mathrm{mg} /$ day group (22.9 [1.5], $p<0.001$ ) and $80-120 \mathrm{mg} /$ day group (22.7 [1.5], $p<0.01)$ in comparison to placebo (15.2 [1.5]). Similar findings were obtained in the adjunctive therapy trial for the lurasidone group in comparison to placebo (23.1 [1.5] versus $17.9[1.6] ; p<0.05)$.

\section{Direct and indirect effects of lurasidone on HRQoL}

The total effect between lurasidone treatment and $\triangle$ Q-LES-Q SF in Model 1 was strong and statistically significant in both trials (Table 5). Relationships tested in Models 2 and 3 were also statistically significant (all $p<0.05$ ). Thus, the full mediation model (Model 4) was analyzed and tested to determine if there was an independent effect of treatment on $\Delta \mathrm{Q}$ LES-Q SF after accounting for the variance explained by $\triangle$ MADRS.

As shown in Fig. 1a and b (for monotherapy and adjunctive therapy, respectively), lurasidone treatment predicted improvement in MADRS (monotherapy $=\beta_{2}=-0.44$;

Table 1 Patient demographic characteristics at baseline

\begin{tabular}{|c|c|c|c|c|c|}
\hline \multirow[t]{2}{*}{ Variable } & \multicolumn{3}{|l|}{ Monotherapy } & \multicolumn{2}{|c|}{ Adjunctive therapy } \\
\hline & $\begin{array}{l}\text { Lurasidone } 20-60 \mathrm{mg} / \text { day } \\
(N=161)\end{array}$ & $\begin{array}{l}\text { Lurasidone } 80-120 \mathrm{mg} / \text { day } \\
(N=162)\end{array}$ & $\begin{array}{l}\text { Placebo } \\
(N=162)\end{array}$ & $\begin{array}{l}\text { Lurasidone } \\
(N=179)\end{array}$ & $\begin{array}{l}\text { Placebo } \\
(N=161)\end{array}$ \\
\hline Age, mean (SD) & $41.3(12.3)$ & $42.0(12.4)$ & $41.2(12.4)$ & $41.0(11.5)$ & $42.6(11.8)$ \\
\hline Male, n (\%) & 70 (43.5 \%) & $64(39.5 \%)$ & 75 (46.3 \%) & 93 (52.0 \%) & 85 (52.8 \%) \\
\hline White, n (\%) & $107(66.5 \%)$ & $106(65.4 \%)$ & $107(66.0 \%)$ & $108(60.3 \%)$ & $102(63.4 \%)$ \\
\hline $\begin{array}{l}\text { Baseline Q-LES-Q SF Percentage Maximum Score, } \\
\text { mean (SD) }\end{array}$ & $51.3(20.8)$ & $52.1(20.0)$ & $45.5(20.5)$ & $55.9(19.6)$ & $50.4(20.6)$ \\
\hline Baseline MADRS Total Score, mean (SD) & $30.3(5.0)$ & $30.6(4.9)$ & $30.5(4.9)$ & $30.5(5.3)$ & $30.7(4.8)$ \\
\hline
\end{tabular}


Table 2 Mean change for Q-LES-Q SF percentage maximum scores from baseline to 6 weeks: monotherapy (Lurasidone 20-60 mg/ day and Lurasidone $80-120 \mathrm{mg} /$ day)

\begin{tabular}{|c|c|c|c|c|c|c|}
\hline & \multicolumn{3}{|c|}{ Lurasidone 20-60 mg/day } & \multicolumn{3}{|c|}{ Lurasidone $80-120$ mg/day } \\
\hline & N Mean (SD) & N Mean Change (SD) & $P$ value & N Mean (SD) & N Mean Change (SD) & $P$ value \\
\hline \multicolumn{7}{|c|}{ Q-LES-Q SF } \\
\hline Baseline & $15733.8(13.7)$ & - & & $16033.5(13.0)$ & - & \\
\hline 6-Weeks & 12355.9 (19.4) & $12021.9(16.0)$ & $<.0001$ & $12156.8(18.1)$ & $12023.0(17.7)$ & $<.0001$ \\
\hline
\end{tabular}

All $p$-values for Q-LES-Q SF outcomes are based on $t$-tests comparing differences from baseline to 6 weeks within treatment group

adjunctive therapy: $\beta_{2}=-0.34$ ), which subsequently predicted improvement in HRQoL (monotherapy: $\beta_{3}=-0.73$; adjunctive therapy: $\left.\beta_{3}=-0.75\right)$. However, the direct effect of treatment on $\triangle$ Q-LES-Q SF after accounting for $\triangle$ MADRS did not remain statistically significant in either study, as the direct effect estimate was considered small (monotherapy: $\beta_{4}=0.11, p=0.13$ ) to near 0 (adjunctive therapy: $\left.\beta_{4}=0.02, p=0.77\right)$. This finding is reflected in the indirect effects, estimated as 0.32 in the monotherapy trial $(p<0.001)$ and $0.26(p<0.01)$ in the adjunctive therapy trial. The percentage of variance accounted for by the indirect effect through $\triangle$ MADRS was $73 \%$ and $91 \%$, respectively. For both studies, the full mediation models with direct and indirect effects explained the majority of the variability in $\triangle \mathrm{Q}-\mathrm{LES}-\mathrm{Q}$ SF (monotherapy: $R^{2}=60.5 \%$; adjunctive therapy: $R^{2}=66.2 \%$ ). The findings from this analysis provide evidence for partial mediation in the monotherapy trial and almost complete mediation in the adjunctive therapy trial.

\section{Discussion}

In this post-hoc analysis of two 6-week, randomized, placebo-controlled clinical trials conducted to assess the efficacy of lurasidone on HRQoL in patients with bipolar depression, lurasidone performed significantly better in comparison to placebo in improving patient-reported HRQoL. In analyses of lurasidone as a monotherapy and adjunctive therapy to lithium or valproate, similar adjusted change in the Q-LES-Q SF was evidenced for all treatment groups receiving lurasidone. The overall findings provide evidence that lurasidone performs similarly as a monotherapy and as an adjunctive therapy, at a range of dosage levels, in improving self-reported HRQoL in patients with bipolar depression.
While morbidity and mortality is high in patients with bipolar depression and HRQoL is often greatly compromised, improvement in HRQoL in clinical treatment trials for bipolar depression is often understudied. However, the relatively few studies that have been published provide support for the assertion that treatment can improve HRQoL in patients with bipolar depression. Using the Medical Outcomes Study 36-Item ShortForm Health Survey (SF-36) as a measure of HRQoL in an 8-week randomized, controlled trial of olanzapine or olanzapine-fluoxetine combination, Shi and colleagues [33] found that patients receiving either olanzapine or olanzapine-fluoxetine reported significantly greater improvement on the mental and physical component scores of the SF-36. More recently, Endicott and colleagues [34] conducted a secondary analysis of two 8-week randomized, controlled clinical trials of quetiapine. Using a mixed model for repeated measures analysis, they found that patients taking 300 and $600 \mathrm{mg} /$ day significantly improved on the Q-LESQ SF with adjusted least squares mean (SE) change scores of 10.89 (0.59) and 12.14 (0.62), respectively, by Week 8. These findings parallel earlier work where QLES-Q SF scores were significantly improved in patients taking quetiapine in comparison to placebo at Week 4 and Week 8 [35]. The present study demonstrated that in controlled analyses, lurasidone was even more effective in improving HRQoL as measured by the Q-LES-Q SF, where mean (SD) change scores were 22.5 (16.9) and 23.3 (18.7) in the monotherapy and adjunctive therapy studies, respectively.

The HRQoL improvement in patients receiving lurasidone parallel the reduction in MADRS depression scores in this sample [23, 24]. Specifically, both as a monotherapy

Table 3 Mean change for Q-LES-Q SF percentage maximum scores from baseline to 6 weeks: monotherapy (combined lurasidone treatment groups and placebo)

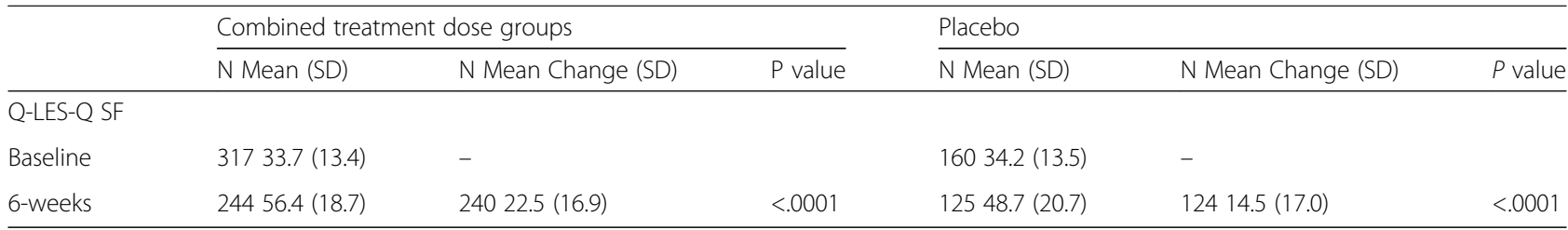

All $p$-values for Q-LES-Q SF outcomes are based on $t$-tests comparing differences from baseline to 6 weeks within treatment group 
Table 4 Mean Change for Q-LES-Q SF percentage maximum scores from baseline to 6 weeks: adjunctive therapy (Lurasidone 20-120 mg/day and Placebo)

\begin{tabular}{|c|c|c|c|c|c|c|}
\hline & \multicolumn{3}{|c|}{ Lurasidone 20-120 mg/day } & \multicolumn{3}{|l|}{ Placebo } \\
\hline & N Mean (SD) & N Mean Change (SD) & $P$ value & N Mean (SD) & N Mean Change (SD) & $P$ value \\
\hline \multicolumn{7}{|c|}{ Q-LES-Q SF } \\
\hline Baseline & $17736.1(14.3)$ & - & & $15935.7(13.5)$ & - & \\
\hline 6-Weeks & $14258.4(181)$ & $14123.3(18.7)$ & $<.0001$ & $13552.7(19.9)$ & $13417.9(20.2)$ & $<.0001$ \\
\hline
\end{tabular}

All p-values for Q-LES-Q SF outcomes are based on $t$-tests comparing differences from baseline to 6 weeks within treatment group

and an adjunctive therapy, lurasidone treatment had a significantly greater effect in reducing depression over 6 weeks in comparison to placebo as measured by the MADRS and the Clinical Global Impressions-Bipolar scale. Path analysis revealed that MADRS total score improvement was a partial (monotherapy) and almostcomplete mediator (adjunctive therapy) on the relationship between treatment and improvement in the Q-LESQ SF. Thus, improvement in HRQoL in lurasidonetreated patients largely occurred through improvements in depression symptoms. This finding of partial (rather than full) mediation in the monotherapy trial could be due to larger mean Q-LES-Q SF improvement resulting from lurasidone administered as a monotherapy in comparison to an adjunctive therapy. These findings are supported by previous investigations comparing the effects of monotherapy and combination therapy on HRQoL using the investigator-rated global assessment of functioning (GAF) in patients with bipolar disorder $[36,37]$. However, future research assessing the incremental benefit of monotherapy over adjunctive therapy on HRQoL improvement is needed in patients with bipolar depression.

This knowledge of both the direct and indirect effects of lurasidone treatment on improvement in HRQoL provides a more holistic view of the complex effect of treatment on the magnitude and level of improvement in how a patient feels or functions. Whereas the primary goal of treatment for patients with bipolar depression is often the alleviation of depression symptoms, improved HRQoL is often critical to patients and their clinicians [4], and could potentially shape long-term recovery. For example, improved HRQoL has been associated with greater adherence to treatment [38], which has been

Table 5 Path analysis of models 1, 2, and 3 for monotherapy and adjunctive therapy trials

\begin{tabular}{lll}
\hline Model & Monotherapy $\beta$ ( $p$-value) & Adjunctive therapy $\beta$ ( $p$-value) \\
\hline Model 1 & $\beta_{1}=0.45(p<0.001)$ & $\beta_{1}=0.28(p<0.05)$ \\
Model 2 & $\beta_{2}=-0.41(p<0.01)$ & $\beta_{2}=-0.36(p<0.01)$ \\
Model 3 & $\beta_{3}=-0.77(p<0.001)$ & $\beta_{3}=-0.75(p<0.001)$ \\
\hline
\end{tabular}

shown to be a great barrier to effective long-term treatment in patients with bipolar depression [39].

The results of the present study are supported by previous investigations that have demonstrated that a strong relationship exists between a depressed clinical state and reduced HRQoL in patients with bipolar disorder [34, 40-42]. For example, using data from the Systematic Treatment Enhancement Program for Bipolar Disorder (STEP-BD) multicenter trial, researchers sought to determine the unique contribution of clinical states (e.g., depression, mania/hypomania, mixed) in relation to HRQoL. Bipolar patients with depression had significantly lower HRQoL scores in comparison to recovered, recovering, and patients with mania/hypomania, controlling for sociodemographic and health characteristics. Similar conclusions were drawn by Saarni et al. [43] and Vojta [41], concluding that in bipolar patients, depressive symptoms are the strongest predictors of reduced HRQoL. Also using the Q-LES-Q SF and MADRS to assess HRQoL and depression, respectively, Endicott [34] found that improvement in HRQoL across an 8-week period was most strongly correlated with improvements in MADRS scores. In the current study, we expanded on these analyses using path analysis, finding that not only is change in these constructs correlated across time, but that change in MADRS is predictive of change in Q-LES-Q SF, thus providing an assessment of the mediating effect of depression on the treatment-HRQoL relationship.

Several study limitations should be noted. The study duration was only 6 weeks, and a longitudinal study is needed to determine if patients maintain the demonstrated improvement or continue to improve over a longer period of time. However, a 6-week trial length is a standard for acute depression clinical trials $[44,45]$. In addition, HRQoL was assessed based on patient-reported data, and not direct observation of patient behavior or functioning, and the assessment was conducted with only one assessment of HRQoL. The findings from the current study could be different if an alternative PRO of HRQoL was selected. Finally, we used path analysis in a limited sample to determine if depression mediated the treatmentHRQoL relationship. A replication of these analyses in a 

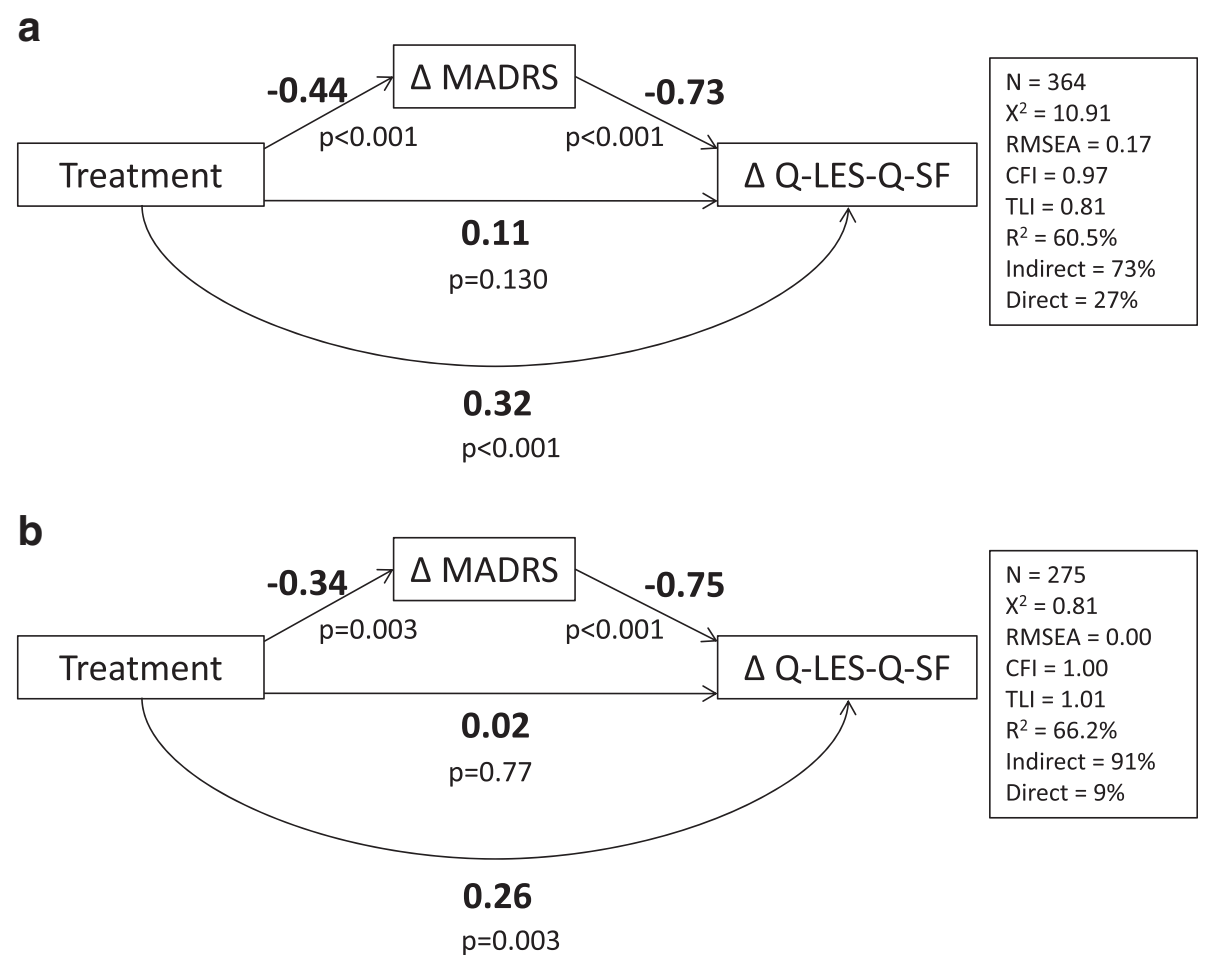

$\mathrm{N}=275$

$X^{2}=0.81$

RMSEA $=0.00$

$\mathrm{CFI}=1.00$

$\mathrm{TLI}=1.01$

$\mathrm{R}^{2}=66.2 \%$

Indirect $=91 \%$

Direct $=9 \%$

$p=0.003$

Fig. 1 a Path analysis model 4: Monotherapy. b Path analysis model 4: adjunctive therapy

larger population is needed to confirm our findings. Although mediation analysis allows for the interpretation of causal relationships from correlational data, additional research using a causal study design is necessary to validate these findings.

\section{Conclusions}

In summary, patients with bipolar depression have greatly impaired HRQoL. Lurasidone as a monotherapy (20-60 mg/day and $80-120 \mathrm{mg} /$ day) and adjunctive to lithium or valproate (20-120 mg/day) was efficacious in improving HRQoL in this study. Change in HRQoL is largely mediated by improvement in depression symptoms. These findings underscore the significance of treatment effectiveness in reduction of depressive symptoms, which in turn result in improvement of HRQoL in patients with bipolar depression.

\section{Abbreviations}

ANCOVA: Analysis of covariance; CFI: Comparative Fit Index; DSM-IV-TR: Diagnostic and Statistical Manual-IV-TR; GAF: Global assessment of functioning; HRQoL: Healthrelated quality of life; ITT: Intention-to-treat; $\triangle M A D R S$ : Mean change in MADRS total score; MADRS: Montgomery-Asberg Depression Rating Scale; PRO: Patient-reported outcome; $\triangle \mathrm{Q}-\mathrm{LES}-\mathrm{Q}$ SF: Mean change in Q-LES-Q SF percentage maximum scores; Q-LES-Q SF: Quality of life enjoyment and satisfaction questionnaire short form; RMSEA: Root mean square error of approximation; SE: Standard errors; STEP-BD: Systematic treatment enhancement program for bipolar disorder; TLI: Tucker lewis index; YMRS: Young mania rating scale.

\section{Acknowledgements}

The authors thank the participants of this study, as well as the members of the Lurasidone Bipolar Disorder Study Group (Czech Republic: Drs. J. Drahozal, E. Herman, M. Klabusayova, and Z. Solle; France: Dr. M. Zins Ritter; India: Drs. M. Chudgar, H. Gandhi, M. Gowda, V. Indla, U. Nagapurkar, J. Trivedi, P. Thatikonda, M. Vaishnav, and R. Yadav; Romania: Drs. M.G. Badescu, D. Cozman, G. Oros, and D. Vasile; Russia: Drs. M. Ivanov, V. Kozlovsky, V. Tochilov, and V. Vid; South Africa: Drs. K. Botha, G. Jordaan, L. Nel, J. Schronen; Ukraine: Drs. V. Abramov, V. Bitenskyy, S. Rymsha, A. Skrypnikov, and V. Verbenko; United States: Drs. S. Aaronson, M. Alam, S. Atkinson, P. Bhatia, R. Brenner, J. Calabrese, A. Cutler, G. Dempsey, D. Garcia, E. Gfeller, H. Hassman, R. Knapp, R. Manning, J. Miller, N. Rosenthal, R. Hidaldo, T. Tran-Johnson, N. Vatakis, D. Walling, R. Weisler, K. Yadalam, M. Allen, J. Kunovac, and S. Potkin).

\section{Funding}

The study was sponsored by Sunovion Pharmaceuticals, Inc.

\section{Availability of data and materials}

The data is confidential clinical trial data, and will not be shared.

\section{Authors' contributions}

AP and AL participated in the original clinical trial study design and conduct, as well as the analysis of data used for the present secondary data analysis. In the present study, they participated in the conceptualization of the study design and interpretation of study findings. KR, EDB, and KW participated in the study design, interpretation of statistical analyses, and manuscript preparation. DN-M helped to draft the manuscript. All authors read and approved the final manuscript.

\section{Competing interests}

Krithika Rajagopalan, Daisy Ng-Mak, Andrei Pikalov, and Antony Loebel are employees of Sunovion Pharmaceuticals Inc. Elizabeth Dansie Bacci and Kathy Wyrwich are employees of Evidera. 


\section{Consent to publish}

Not applicable.

\section{Ethics and consent to participate}

List of IRB/ECs for NCT00868699

- CEC

- Cedars-Sinai Medical Center

- Copernicus Group

- Comisia Nationala de Etica pentru Studiul Clinic al Medicamentului

- Cosmos Independent Ethics Committee

- CPP OUEST IV

- Ethics Committee MITR Hospital

- Ethics Committee-Sheth V.S. General Hospital

- Eticka komise BIALBI, s.r.o

- Eticka komise pro multicentricka kinicka hodnocen FN Motol

- IBIOME Independent Ethics Committee

- Independent Ethics Committee

- Institutional Ethics Committee

- Komisja Bioetyczna

- Landesamt fur Gesundheit und Soziales Berlin

- LEC at SPSRI named after V.M. Bekhterev

- MetroHealth Institutional Review Board

- Ministry of Health of Ukraine

- North Maharashtra Ethics Committee

- Pharma-Ethics

- Spandana Ethics Committee

- Stanford University IRB

- State Pharmacological Center

- University of CA - Irvine

- University of Cape Town

- University Hospitals Case Medical Center IRB

- VIMHANS Hospital Ethic Committee

- Western Institutional Review Board

List of IRB/ECs for NCT00868452

- CEC

- Central Ethics Commission

- Copernicus Group

- Comisia Nationala de Etica pentru Studiul Clinic al Medicamentului

- CPP OUEST IV

- The Ethics Committee

- Ethics Committee MITR Hospital

- Ethics Committee-V.S. General Hospital

- Eticka komise BIALBI, s.ro

- Eticka komise NZZ Clinitrial, s.r.o.

- Eticka komise pro multicentricka kinicka hodnocen FN Motol

- IBIOME Independent Ethics Committee

- Independent Ethics Committee

- Institutional Ethics Committee

- LEC at SPSRI named after V.M. Bekhterev

- MetroHealth Institutional Review Board

- North Maharashtra Ethics Committee

- Pharma-Ethics

- Spandana Ethics Committee

- State Pharmacological Center

- University of CA - Irvine

- University Hospitals Case Medical Center IRB

- VIMHANS Hospital Ethic Committee

- Western Institutional Review Board

\section{Author details}

'Sunovion Pharmaceuticals Inc., Marlborough, MA, USA. ${ }^{2}$ Evidera, Seattle, WA, USA. ${ }^{3}$ Evidera, Bethesda, MD, USA.

\section{Received: 24 June 2015 Accepted: 13 May 2016}

Published online: 23 May 2016

\section{References}

1. Merikangas KR, Akiskal HS, Angst J, Greenberg PE, Hirschfeld RM, Petukhova M, et al. Lifetime and 12-month prevalence of bipolar spectrum disorder in the National Comorbidity Survey replication. Archives of general psychiatry. 2007; 64(5):543-52. doi:10.1001/archpsyc.64.5.543.
2. Merikangas KR, Jin R, He JP, Kessler RC, Lee S, Sampson NA, et al. Prevalence and correlates of bipolar spectrum disorder in the world mental health survey initiative. Archives of general psychiatry. 2011;68(3):241-51. doi:10.1001/archgenpsychiatry.2011.12.

3. World Health Organization. Global Burden of Disease: 2004 Update. Geneva, Switzerland: World Health Organization; 2008.

4. Michalak EE, Murray G, Young AH, Lam RW. Burden of bipolar depression: impact of disorder and medications on quality of life. CNS drugs. 2008; 22(5):389-406

5. Fagiolini A, Coluccia A, Maina G, Forgione RN, Goracci A, Cuomo A, et al. Diagnosis, epidemiology and management of mixed states in bipolar disorder. CNS drugs. 2015;29(9):725-40. doi:10.1007/s40263-015-0275-6.

6. Rosa AR, Reinares M, Michalak EE, Bonnin CM, Sole B, Franco C, et al. Functional impairment and disability across mood states in bipolar disorder. Value in health. 2010;13(8):984-8. doi:10.1111/j.1524-4733.2010.00768.x.

7. Malhi GS, Ivanovski B, Hadzi-Pavlovic D, Mitchell PB, Vieta E, Sachdev P. Neuropsychological deficits and functional impairment in bipolar depression, hypomania and euthymia. Bipolar Disord. 2007;9(1-2):114-25. doi:10.1111/j. 1399-5618.2007.00324.x.

8. Miller S, Dell'Osso B, Ketter TA. The prevalence and burden of bipolar depression. J Affect Disord. 2014;169 Suppl 1:S3-11. doi:10.1016/S0165-0327(14)70003-5.

9. Moreno C, Hasin DS, Arango C, Oquendo MA, Vieta E, Liu S, et al. Depression in bipolar disorder versus major depressive disorder: results from the National Epidemiologic Survey on Alcohol and Related Conditions. Bipolar Disord. 2012; 14(3):271-82. doi:10.1111/j.1399-5618.2012.01009.x.

10. van der Voort TY, Seldenrijk A, van Meijel B, Goossens PJ, Beekman AT, Penninx BW, et al. Functional versus syndromal recovery in patients with major depressive disorder and bipolar disorder. J Clin Psychiatry. 2015;76(6): e809-14. doi:10.4088/JCP.14m09548.

11. Montgomery SA, Asberg M. A new depression scale designed to be sensitive to change. Br J Psychiatry. 1979;134:382-9.

12. Keck Jr PE. Defining and improving response to treatment in patients with bipolar disorder. J Clin Psychiatry. 2004;65 Suppl 15:25-9.

13. Miklowitz DJ, Otto MW, Frank E, Reilly-Harrington NA, Kogan JN, Sachs GS, et al. Intensive psychosocial intervention enhances functioning in patients with bipolar depression: results from a 9-month randomized controlled trial. Am J Psychiatry. 2007;164(9):1340-7. doi:10.1176/appi.ajp.2007.07020311.

14. Piccinni A, Catena M, Del Debbio A, Marazziti D, Monje C, Schiavi E, et al. Health-related quality of life and functioning in remitted bipolar I outpatients. Compr Psychiatry. 2007:48(4):323-8. doi:10.1016/j.comppsych.2006.12.007.

15. Rubio JM, Olfson M, Villegas L, Perez-Fuentes G, Wang S, Blanco C. Quality of life following remission of mental disorders: findings from the National Epidemiologic Survey on Alcohol and Related Conditions. J Clin Psychiatry. 2013;74(5):e445-50. doi:10.4088/JCP.12m08269.

16. Harvey PD. Defining and achieving recovery from bipolar disorder. J Clin Psychiatry. 2006;67 Suppl 9:14-8. discussion 36-42.

17. Michalak EE, Yatham LN, Lam RW. Quality of life in bipolar disorder: a review of the literature. Health Qual Life Outcomes. 2005;3:72. doi:10.1186/1477-7525-3-72.

18. Revicki DA, Matza LS, Flood E, Lloyd A. Bipolar disorder and health-related quality of life: review of burden of disease and clinical trials. PharmacoEcon. 2005:23(6):583-94.

19. IsHak WW, Brown K, Aye SS, Kahloon M, Mobaraki S, Hanna R. Health-related quality of life in bipolar disorder. Bipolar Disord. 2012;14(1):6-18. doi:10.1111/j. 1399-5618.2011.00969.x

20. Tohen M, Vieta E, Calabrese J, Ketter TA, Sachs G, Bowden C, et al. Efficacy of olanzapine and olanzapine-fluoxetine combination in the treatment of bipolar I depression. Arch Gen Psychiatry. 2003:60(11):1079-88. doi:10.1001/ archpsyc.60.11.1079.

21. Sanford M, Keating GM. Quetiapine: a review of its use in the management of bipolar depression. CNS drugs. 2012;26(5):435-60. doi:10.2165/11203840$000000000-00000$

22. Bogart GT, Chavez B. Safety and efficacy of quetiapine in bipolar depression. Ann Pharmacother. 2009:43(11):1848-56. doi:10.1345/aph.1M193.

23. Loebel A, Cucchiaro J, Silva R, Kroger H, Sarma K, Xu J, et al. Lurasidone as adjunctive therapy with lithium or valproate for the treatment of bipolar I depression: A randomized, double-blind, placebo-controlled study. Am J Psychiatry. 2014a;171(2):169-77.

24. Loebel A, Cucchiaro J, Silva R, Kroger H, Sarma K, Xu J, et al. Lurasidone monotherapy in the treatment of bipolar I depression: A randomized double-blind, placebo-controlled study. Am J Psychiatry. 2014b;171(2):160-8. 
25. Sheehan DV, Lecrubier $Y$, Sheehan KH, Amorim P, Janavs J, Weiller E, et al. The Mini-International Neuropsychiatric Interview (M.I.N.I.): the development and validation of a structured diagnostic psychiatric interview for DSM-IV and ICD-10. J Clin Psychiatry. 1998;59 Suppl 20:22-33. quiz 4-57.

26. Sachs GS. Strategies for improving treatment of bipolar disorder: integration of measurement and management. Acta Psychiatrica Scandinavica Supplementum. 2004;422:7-17. doi:10.1111/j.1600-0447.2004.00409.x.

27. Endicott J, Nee J, Harrison W, Blumenthal R. Quality of life enjoyment and satisfaction questionnaire: a new measure. Psychopharmacol Bull. 1993;29(2):321-6.

28. Baron RM, Kenny DA. The moderator-mediator variable distinction in social psychological research: conceptual, strategic, and statistical considerations. J Pers Soc Psychol. 1986;51(6):1173-82.

29. Muthén LK, Muthén BO. Mplus User's Guide. 7th ed. Los Angeles, CA: Muthén \& Muthén; 1998-2013.

30. Kenny DA. Mediation. 2014. http://davidakenny.net/cm/mediate.htm. Accessed 28 Feb 2014

31. MacCallum RC, Browne MW, Sugawara HM. Power analysis and determination of sample size for covariance structure modeling. Psychol Methods. 1996;:130-49.

32. Hu L, Bentler PM. Cutoff criteria for fit indexes in covariance structure analysis. Conventional criteria versus new alternatives. Structural Equation Modeling. 1999;6:1-55.

33. Shi L, Namjoshi MA, Swindle R, Yu X, Risser R, Baker RW, et al. Effects of olanzapine alone and olanzapine/fluoxetine combination on health-related quality of life in patients with bipolar depression: secondary analyses of a double-blind, placebo-controlled, randomized clinical trial. Clin Ther. 2004; 26(1):125-34.

34. Endicott J, Paulsson B, Gustafsson U, Schioler H, Hassan M. Quetiapine monotherapy in the treatment of depressive episodes of bipolar I and II disorder: Improvements in quality of life and quality of sleep. J Affect Disord. 2008;111(2-3):306-19. doi:10.1016/j.jad.2008.06.019.

35. Endicott J, Rajagopalan K, Minkwitz M, Macfadden W, Bolder Study Group. A randomized, double-blind, placebo-controlled study of quetiapine in the treatment of bipolar I and II depression: improvements in quality of life. Int Clin Psychopharmacol. 2007;22(1):29-37. doi:10.1097YIC.0b013e32801035a5.

36. Goldberg JF, Harrow M, Leon AC. Lithium treatment of bipolar affective disorders under naturalistic followup conditions. Psychopharmacol Bull. 1996;32(1):47-54

37. Ghaemi SN, Goodwin FK. Gabapentin treatment of the non-refractory bipolar spectrum: an open case series. J Affect Disord. 2001;65(2):167-71.

38. Nunes MI. The relationship between quality of life and adherence to treatment. J Clin Psychiatry. 2005;66 Suppl 1:24-9.

39. Velligan DI, Weiden PJ, Sajatovic M, Scott J, Carpenter D, Ross R, et al. The expert consensus guideline series: adherence problems in patients with serious and persistent mental illness. J Clin Psychiatry. 2009;70 Suppl 4:1-46. quiz 7-8.

40. Zhang H, Wisniewski SR, Bauer MS, Sachs GS, Thase ME, Systematic Treatment Enhancement Program for Bipolar Disorder Investigators. Comparisons of perceived quality of life across clinical states in bipolar disorder: data from the first 2000 Systematic Treatment Enhancement Program for Bipolar Disorder (STEP-BD) participants. Compr Psychiatry. 2006; 47(3):161-8. doi:10.1016/j.comppsych.2005.08.001.

41. Vojta C, Kinosian B, Glick H, Altshuler L, Bauer MS. Self-reported quality of life across mood states in bipolar disorder. Compr Psychiatry. 2001;42(3): 190-5. doi:10.1053/comp.2001.23143.

42. Namjoshi MA, Buesching DP. A review of the health-related quality of life literature in bipolar disorder. Qual Life Res. 2001;10(2):105-15.

43. Saarni SI, Viertio S, Perala J, Koskinen S, Lonnqvist J, Suvisaari J. Quality of life of people with schizophrenia, bipolar disorder and other psychotic disorders. Br J Psychiatry. 2010;197(5):386-94. doi:10.1192/bjp.bp.109.076489.

44. Pae CU, Forbes A, Patkar AA. Aripiprazole as adjunctive therapy for patients with major depressive disorder: overview and implications of clinical trial data. CNS drugs. 2011;25(2):109-27. doi:10.2165/11538980-000000000-00000.

45. Berman RM, Marcus RN, Swanink R, McQuade RD, Carson WH, Corey-Lisle PK, et al. The efficacy and safety of aripiprazole as adjunctive therapy in major depressive disorder: a multicenter, randomized, double-blind, placebocontrolled study. J Clin Psychiatry. 2007;68(6):843-53.

\section{Submit your next manuscript to BioMed Central and we will help you at every step:}

- We accept pre-submission inquiries

- Our selector tool helps you to find the most relevant journal

- We provide round the clock customer support

- Convenient online submission

- Thorough peer review

- Inclusion in PubMed and all major indexing services

- Maximum visibility for your research

Submit your manuscript at www.biomedcentral.com/submit
Biomed Central 